\title{
Molecular cloning and characterization of gene coding for $\gamma$-tocopherol methyltransferase from lettuce (Lactuca sativa)
}

\author{
Y.L. Tang, W.W. Ren, L. Zhang and K.X. Tang \\ School of Agriculture and Biology, Plant Biotechnology Research Center, \\ Fudan-SJTU-Nottingham Plant Biotechnology R\&D Center, \\ Shanghai Jiao Tong University, Shanghai, China \\ Corresponding author: K.X. Tang \\ E-mail: kxtang1@yahoo.com / kxtang1@163.com
}

Genet. Mol. Res. 10 (4): 3204-3212 (2011)

Received January 6, 2011

Accepted June 7, 2011

Published December 21, 2011

DOI http://dx.doi.org/10.4238/2011.December.21.2

\begin{abstract}
. $\gamma$-tocopherol methyltransferase is an important ratelimiting enzyme involved in tocopherol biosynthesis. The full-length cDNA encoding $\gamma$-tocopherol methyltransferase (designated as LsTMT) was cloned from Lactuca sativa for the first time by rapid amplification of cDNA ends and characterized by means of quantitative RT-PCR. The full-length cDNA of LsTMT was $1131 \mathrm{bp}$, with an open reading frame of $897 \mathrm{bp}$ encoding a $\gamma$-tocopherol methyltransferase protein of 298 amino acids, with a calculated molecular mass of $33.06 \mathrm{kDa}$ and an isoelectric point of 5.86. Comparative analysis revealed that LsTMT has a close similarity with $\gamma$-TMTs from other plant species. Bioinformatic analysis indicated that $L s T M T$ shares a common evolutionary origin based on sequence similarity and has the closest relationship to $\gamma$-TMT from the sunflower, Helianthus annuus. Based on quantitative RT-PCR analysis, we found that expression of LsTMT is induced and strengthened by oxidative stresses such as strong light and drought. The cloning and characterization of $L S T M T$ will be helpful to further understanding its role
\end{abstract}


in the tocopherol biosynthesis pathway. We consider it to be a candidate gene for metabolic engineering of vitamin $\mathrm{E}$ in vegetable crops.

Key words: Lactuca sativa; $\gamma$-tocopherol methyltransferase; RACE; Quantitative RT-PCR

\section{INTRODUCTION}

Tocochromanols, known as vitamin E, are essential nutrients for human health. Tocochromanols are recognized as a group of compounds with amphipathic properties of eight derivatives and can be classified into two groups by the degree of saturation of their hydrophobic tails. The group characterized by a fully saturated tail belongs to tocopherols; while the tocotrienol group has an unsaturated tail. Both groups are composed of four members differing in the number and position of methyl groups on the aromatic ring, named $\alpha-, \beta-, \gamma-$, and $\delta$-tocopherol, respectively. $\alpha$-tocopherol is a major isoform in most plant tissues except in seeds where $\gamma$-tocopherol is the dominant form.

The main function of tocopherol lies in its fatty acyl chain-breaking activity, which scavenges reactive oxygen species (ROS) resulting from photosynthesis, thus protecting polyunsaturated fatty acid chains (PUFAs) from lipid peroxidation. Increasing evidence suggests that in higher plants, vitamin E may play a protective role in cell membrane systems, thus maintaining the integrity and normal function of the photosynthetic apparatus (Havaux et al., 2005; Collin et al., 2008).

$\gamma$-tocopherol methyltransferase ( $\gamma$-TMT) catalyzes the conversion of $\gamma$-tocopherol into $\alpha$-tocopherol. So far, the $\gamma$-TMT gene has been cloned from Arabidopsis thaliana, Brassica napus, Triticum aestivum, Helianthus annuus, and many other plants. The overexpression of the $\gamma$-TMT gene (mostly from A. thaliana) has been reported in Perilla frutescens, Lactuca sativa, Brassica juncea, Codonopsis lanceolata, and in soybean seed (Van Eenennaam et al., 2003; Cho et al., 2005; Kim et al., 2005; Yusuf and Sarin, 2007; Lee et al., 2008; Seong et al., 2009), which demonstrated the increase of $\alpha$-tocopherol. In this study, the gene encoding $\gamma$-tocopherol methyltransferase, LsTMT, was cloned from $L$. sativa for the first time. Its expression pattern is also analyzed by means of quantitative RT-PCR. The attainment of new genes involved in vitamin E biosynthesis could offer candidate genes for enhancing vitamin $E$ levels of vegetables by transgenic engineering.

\section{MATERIAL AND METHODS}

\section{Plant materials and isolation of RNA}

Lactuca savita was grown at $25^{\circ} \mathrm{C}$ during the day $/ 18^{\circ} \mathrm{C}$ at night in a chamber $(16-\mathrm{h}$ light/8-h dark cycle). Leaves of 3-month-old plants were used for total RNA isolation by using TIANGEN reagent following the manufacturer instruction (TIANGEN, China). After isolation, total RNA was used for cDNA cloning. The quality and concentration of RNA were measured by agarose gel electrophoresis and spectrophotometry before later steps.

\section{Cloning of the full-length cDNA of LsTMT}

Full-length cDNA of LsTMT was cloned by means of rapid amplification of cDNA ends 
(RACE) according to the protocol of the SMART ${ }^{\mathrm{TM}} \mathrm{RACE}$ cDNA Amplification Kit (Clontech, USA). The core fragment of LsTMT was obtained using TMT-corel (5'-TAGTTGATGTC/ TGGGTGTGGT/C-3') and TMT-core2 (5'-TTTACATAATCAGCT/AGTAGAA/GCACC$\left.3^{\prime}\right)$ as the PCR-amplification primers, designed based on two highly conserved amino acid blocks of $\gamma$-TMTs from other species. 5'-RACE-Ready cDNA of LsTMT was synthesized with 5'-CDS Primer A $\left(5^{\prime}-(\mathrm{T})_{25} \mathrm{VN}-3^{\prime}, \mathrm{V}=\mathrm{A}, \mathrm{G}\right.$, or C) and SMARTII ${ }^{\mathrm{TM}} \mathrm{A}$ Oligonucleotide (5'-AAGCAGTGGTATCAACGCAGAGTACGCGGG-3') as primers, and total RNA isolated earlier as the template. Similarly, 3'-RACE-Ready cDNA of $L s G-T M T$ was synthesized with 3'-CDS Primer A (5'-AAGCAGTGGTATCAACGCAGAGTAC $\left.(\mathrm{T})_{30} \mathrm{VN}-3^{\prime}\right)$ as the primer, and total RNA isolated earlier as the template. Using the 3'-RACE cDNA as the template, primer LsTMT3'-GSP (GSP2: 5'-TACCTCCCGAAAAGTCCCTACGCCC-3') designed according to the sequence of the core fragment of $L S T M T$ was applied as the forward primer and Universal Primer A Mix (UPM: 5'-CTAATACGACTCACTATAGGGCAAGCAGTGGTATCAACGCAGA GT-3'), which recognized the SMARTII A Oligonucleotides, as the reverse primer for the 3 '-end cDNA amplification. The PCR amplification was performed as follows: 5 cycles $\left(94^{\circ} \mathrm{C}\right.$ for 30 $\mathrm{s}, 72^{\circ} \mathrm{C}$ for $\left.2 \mathrm{~min}\right), 5$ cycles $\left(94^{\circ} \mathrm{C}\right.$ for $30 \mathrm{~s}, 70^{\circ} \mathrm{C}$ for $30 \mathrm{~s}$ and $72^{\circ} \mathrm{C}$ for $\left.3 \mathrm{~min}\right), 25$ cycles $\left(94^{\circ} \mathrm{C}\right.$ for $30 \mathrm{~s}, 68^{\circ} \mathrm{C}$ for $30 \mathrm{~s}$ and $72^{\circ} \mathrm{C}$ for $2 \mathrm{~min}$ ). Similarly, the $5^{\prime}$-RACE cDNA used as the template, primer LsTMT5'-GSP (GSP1: 5'-CTGGGCGTAGGGACTTTTCGGGAGG-3') that was also designed according to the sequence of the core fragment of $L S T M T$ was applied as the forward primer and UPM as the reverse primer for the 5'-end cDNA amplification. The PCR amplification was performed under the same conditions as used for 5'-RACE above. The two amplified PCR products were purified and cloned into pMD18-T vector (TaKaRa, Japan) for sequencing. By comparing and aligning the sequences of 5'-RACE and 3'-RACE products, the full-length cDNA sequence of the LSTMT gene was obtained and its coding region was subsequently amplified using a pair of primers, TMT-ORF1 (5'-ATGGCGACTGCAGCGGATGAGC-3') and TMT-ORF2 (5'-TTATTCAGGCTTTTTACATG-3').

\section{Bioinformatic analysis}

Sequence alignments, open reading frame (ORF) translation and molecular mass calculation of predicted protein were carried out with Vector NTI Suite 8.0. BLAST (Basic Local Alignment Search Tool) was performed at the NCBI server (http://www.ncbi.nlm.nih.gov/ blast/Blast.cgi), whereas secondary structural analysis of predicted $L s T M T$ was carried out on the website http://www.expasy.org. The phylogenetic analysis of LsTMT and $\gamma$-TMTs from other species was aligned with CLUSTAL W (a multiple sequence alignment program, version 1.82). MEGA (molecular evolutionary genetics analysis, version 2.1) was subsequently used to construct the phylogenetic tree by applying the neighbor-joining method.

\section{Expression pattern analysis}

SYBR Green I chimeric fluorescence quantitative real-time PCR was used to survey the expression pattern of LsTMT under high light and drought stresses. Total RNA was separately isolated from mature leaves of 8-week-old plants of lettuce, which had been treated with high light (light intensity: $1000 \mu \mathrm{mol} \cdot \mathrm{m}^{-2} \cdot \mathrm{s}^{-1}$ ) and drought (no irrigation) for $0,3,6,9,12$, and 15 days (growing conditions: $16-\mathrm{h} \mathrm{light} / 8$-h dark cycle, at $25^{\circ} \mathrm{C}$ during the day $/ 18^{\circ} \mathrm{C}$ at night). 
Each of the 12 RNA samples was then reversely transcribed into cDNA with TMT-RT1 (5'-GCTTGGTGTTCTACCGCTGATTATG-3') and TMT-RT2 (5'-GCTCCTCTTATAGTCTT CCATCCAC-3') as primers, and cDNA as the template. PCR was performed with an approximately 200-bp long product. The PCR protocol was as follows: 1 cycle $\left(95^{\circ} \mathrm{C}\right.$ for $\left.20 \mathrm{~s}\right)$ and 40 cycles $\left(95^{\circ} \mathrm{C}\right.$ for $15 \mathrm{~s}, 60^{\circ} \mathrm{C}$ for $15 \mathrm{~s}$ and $72^{\circ} \mathrm{C}$ for $\left.25 \mathrm{~s}\right)$. And as a control, the ubiquitin gene was amplified following the above-mentioned PCR procedure. The PCR was repeated three times.

\section{RESULTS}

\section{Cloning of the full-length cDNA of $L s T M T$}

By using the above mentioned methods, the full-length cDNA of LsTMT was obtained. The full-length cDNA of LsTMT was $1131 \mathrm{bp}$ long, including 5'- and 3'-untranslated regions, polyA tail, and a 897-bp ORF encoding a 298-amino-acid protein (Figure 1).

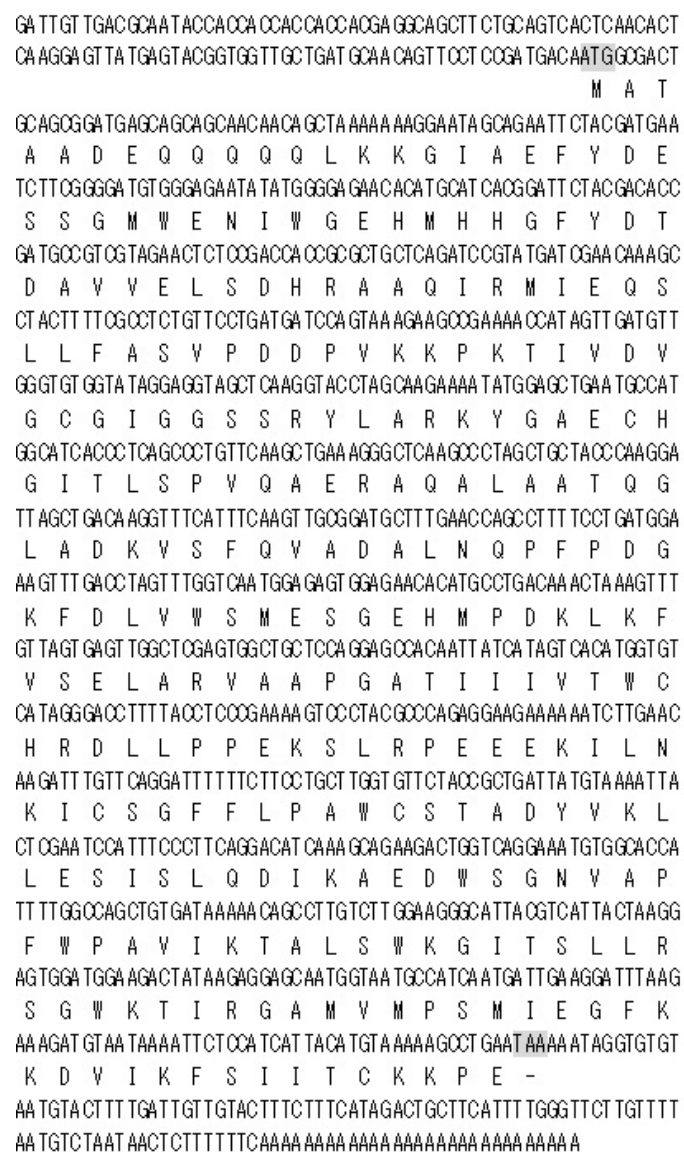

Figure 1. The full-length cDNA and deduced amino acid sequence of $\gamma$-TMT from Lactuca sativa. The start codon (ATG) and the stop codon (TAA) are shaded. 


\section{Bioinformatic analysis}

The deduced LSTMT had a calculated molecular mass of $33.06 \mathrm{kDa}$ and a pI value of 5.86. The most frequent amino acid in deduced $L s T M T$ was Ala (9.7\% by frequency), followed by Leu, Lys and Ser (all 7.7\%). Negatively charged residues (Asp+Glu) constituted $12.75 \%$ of the polypeptide, while positively charged residues (Arg+Lys) constituted $11.07 \%$ of the polypeptide. The protein was computed to have an instability index (II) of 44.64, which classified the protein as unstable (ProtParam, http://us.expasy.org/tools/ protparam.html).

Protein-protein BLAST analysis showed that LsTMT had high homology to $\gamma$-TMTs from other plants (http://www.ncbi.nlm.nih.gov) (Figure 2), such as H. annuus, Solanum tuberosum, T. aestivum, Gossypium hirsutum, P. frutescens, Elaeis oleifera, Zea mays, Hevea brasiliensis, Glycine max, B. napus, A. thaliana, B. oleracea, with similarity of $90,78,76,75,76,72,73,72,77,70,72$, and $71 \%$, respectively.

The secondary structure of LSTMT was analyzed by SOPMA (Geourjon and Deléage, 1995 ) and the result showed that the putative $L s T M T$ peptide contained $54.70 \%$ of $\alpha$ helix, $11.41 \%$ of extended strand, $3.69 \%$ of $\beta$ turn, and $30.20 \%$ of random coil (Figure 3 ). The $\alpha$ helix constituted the majority of the secondary structure, and was the basic element of the $\mathrm{N}$-terminal part, while the extended strand was the primary element of the C-terminal part.

The phylogenetic tree was generated using the present complete $\gamma$-TMT proteins deposited in NCBI. The tree indicated that LsTMT was the most homologous to $\gamma$-TMT of H. annuus (HaTMT) relative to $\gamma$-TMTs from other plants (Figure 4).

\section{Expression profiles of $L s T M T$ under high light and drought}

Real-time RT-PCR was performed to investigate the LsTMT expression pattern under stresses like high light and drought. The results indicated that the expression of LsTMT was strengthened by the two stresses (Figure 5). Expression level of LsTMT gradually increased during 15 days of high light treatment (light intensity: $1000 \mu \mathrm{mol} \cdot \mathrm{m}^{-2} \cdot \mathrm{s}^{-1}$ ). During the session of drought (no irrigation) treatment, expression level of LSTMT peaked after 12 days of the treatment, and then declined remarkably after the peak.

\section{DISCUSSION}

$\gamma$-tocopherol methyltransferase is a crucial enzyme in tocopherol synthesis. In our study, we successfully isolated a $\gamma-T M T$ gene from $L$. sativa for the first time. The deduced amino acid sequence of $L S T M T$ has extensive similarity to its counterparts from other plants. All molecular analyses showed that LsTMT was quite similar in sequence to other $\gamma$-TMTs, especially to that from H. annuus, suggesting that LsTMT belongs to the $\gamma$-TMT family. The isolation and characterization of LsTMT will lead to profound research into its roles involved in tocopherol biosynthesis at the molecular level and provide useful candidate genes for enhancing the content of vitamin $\mathrm{E}$ in food by transgenic technique.

Up to now, many $\gamma$-TMT genes from different species have been found. A phylogenetic tree of $\gamma$-TMTs from different plant species was constructed. LsTMT was clustered with that from H. annuus (HaTMT), since they both belong to Compositae species (Figure 4). 

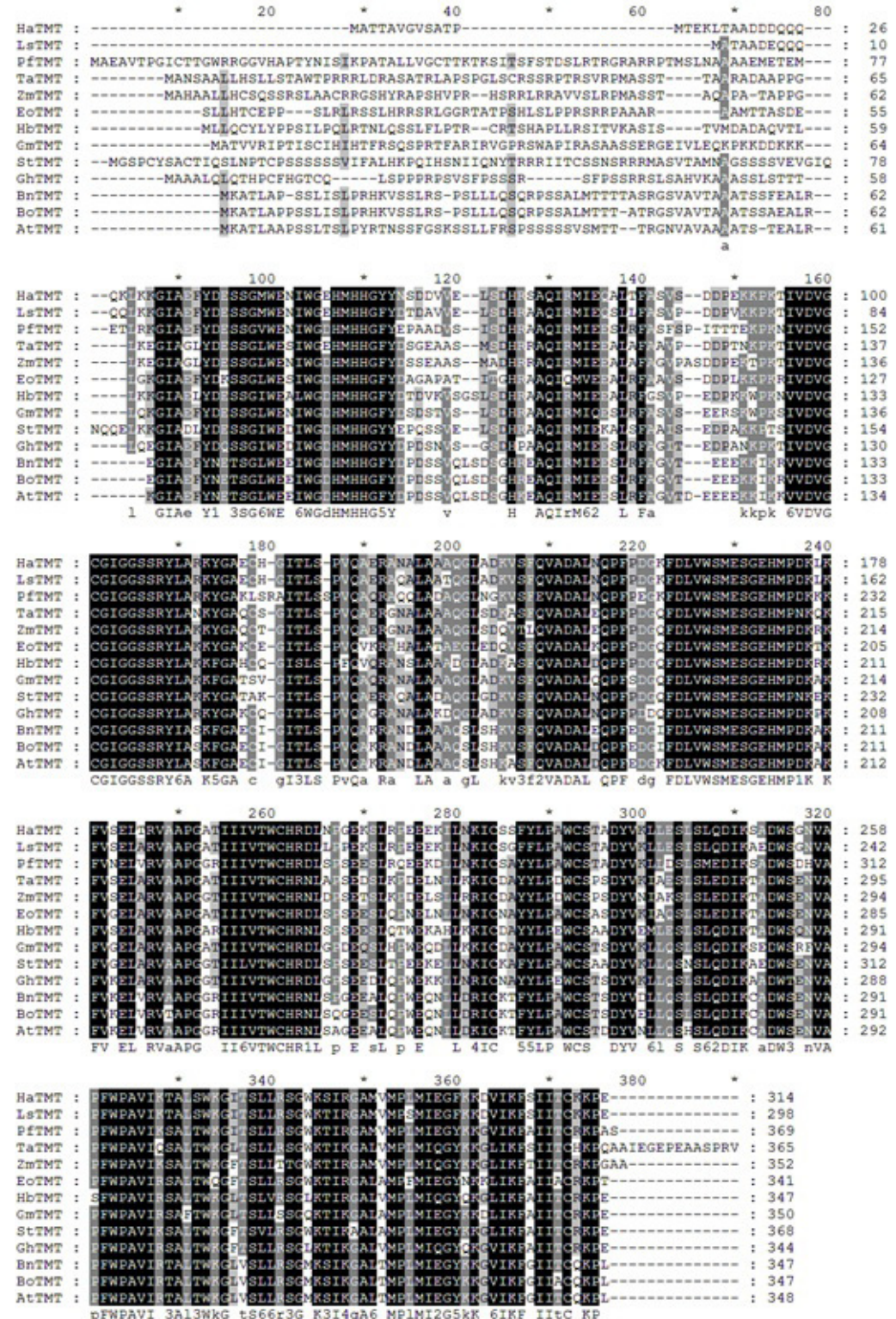

Figure 2. Multiple alignment of the deduced amino acid sequences of LsTMT and other $\gamma$-TMTs. Amino acid sequences were aligned as follows: LsTMT, HaTMT (Helianthus annuus, ABB52798), StTMT (Solanum tuberosum, ABE41795), TaTMT (Triticum aestivum, CAI77219), GhTMT (Gossypium hirsutum, ABE41798), PfTMT (Perilla frutescens, AAL36933), EoTMT (Elaeis oleifera, ABS76142), ZmTMT (Zea mays, ACG34926), HbTMT (Hevea brasiliensis, BAH10645), GmTMT (Glycine max, AAX63899), BnTMT (Brassica napus, ACD03288), AtTMT (Arabidopsis thaliana, AAM64696), BoTMT (Brassica oleracea, AAO13806). Completely identical amino acids are indicated with capital letters against a black background. Less conserved amino acids are indicated with capital letters against dark-gray or relatively light-gray background. Non-conserved amino acids are indicated with black capital letters against a white background. Consensus sequence (the average sequence) is shown below the aligned sequences. 


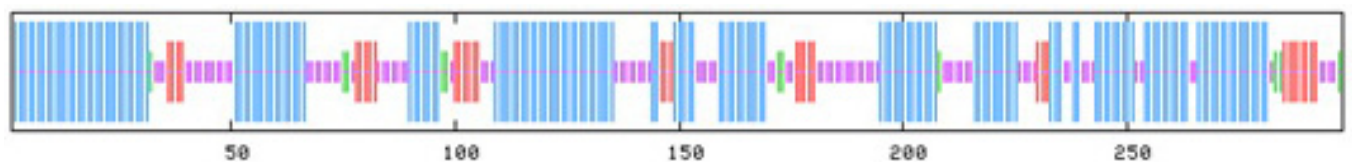

Figure 3. The secondary structure of LsTMT. Alpha helix, extended strand, beta turn, and random coil are indicated, respectively, with the longest, the second longest, the second shortest, and the shortest vertical lines.

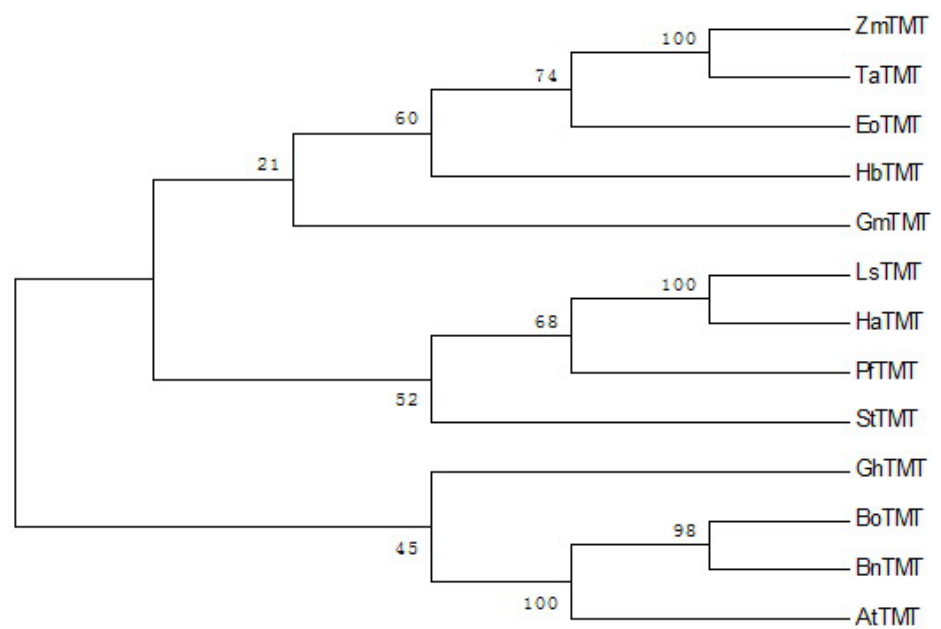

Figure 4. Neighbor-joining evolutionary tree of $\gamma$-TMTs. The tree was constructed with $L s T M T$ and the sequences from NCBI as follows: HaTMT (Helianthus annuus, ABB52798), StTMT (Solanum tuberosum, ABE41795), TaTMT (Triticum aestivum, CAI77219), GhTMT (Gossypium hirsutum, ABE41798), PfTMT (Perilla frutescens, AAL36933), EoTMT (Elaeis oleifera, ABS76142), ZmTMT (Zea mays, ACG34926), HbTMT (Hevea brasiliensis, BAH10645), GmTMT (Glycine max, AAX63899), BnTMT (Brassica napus, ACD03288), AtTMT (Arabidopsis thaliana, AAM64696), BoTMT (Brassica oleracea, AAO13806). The numbers on the branches represent bootstrap support for 1000 replicates.

ROS generated during photosynthesis and metabolism under oxidative stress can induce lipid peroxidation in plant cells and $\alpha$-tocopherol content increases in response to a variety of abiotic stresses (Noctor, 2006) to prevent probable oxidative damage to lipid components by scavenging lipid peroxy radicals in the green parts of plants (Hare et al., 1998; Igamberdiev and Hill, 2004; Kiffin et al., 2006).

There is much evidence that tocopherol content correlates positively with tolerance to low temperature, high light, water deficit, and salt stress in different plants (YamaguchiShinozaki and Shinozaki, 1994; Munne-Bosch et al., 1999; Guo et al., 2006). Induction of the expression of genes, including $\gamma-T M T$, in the tocopherol pathway promotes the accumulation of tocopherols under such stresses that may cause oxidative damage to a certain extent.

A previous study has shown that $\gamma$-TMT-silenced plants have strongly decreased salt tolerance (Abbasi et al., 2007). And overexpressing the $\gamma$-TMT gene greatly increased $\alpha$-tocopherol level and enhanced tolerance to the stress induced by salt, heavy metal and osmoticum in B. juncea (Yusuf et al., 2010).

The quantitative RT-PCR result in our study demonstrates that the expression level of 
the deduced $\gamma$-TMT gene from lettuce can be increased by high light and drought treatment on the transcriptional level (Figure 5), suggesting that more $\gamma$-tocopherol methyltransferases were generated for the synthesis of $\alpha$-tocopherol, which may function as an antioxidant.
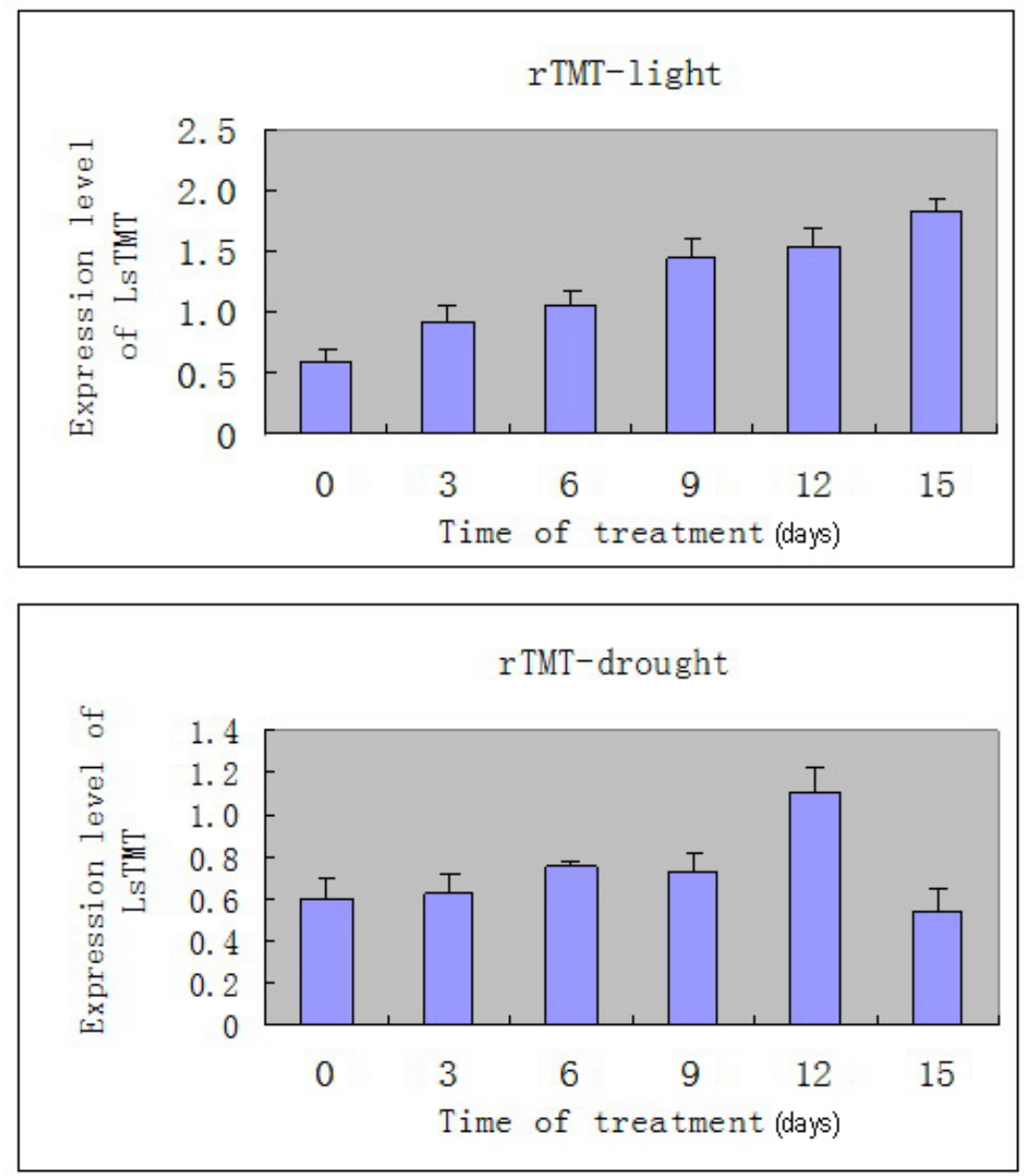

Figure 5. Real-time RT-PCR analysis of $L s T M T$ expression. A. Expression profile of LsTMT under high light treatment for 15 days. B. Expression profile of LsTMT under drought treatment for 15 days. The RT-PCR experiment was repeated three times.

In the drought treatment, the expression level of $\gamma-T M T$ declined markedly at the end, maybe as the plant was dying after an extended period of time without water, which severely affected its metabolism. In an earlier report, the expression level of several genes encoding ROSscavenging enzymes was found to be upregulated in the $\gamma$-TMT-overexpressing deoduck plants (Seong et al., 2009). Our result, along with the above-mentioned reports, seems to indicate that $\gamma$-TMT can help to protect cells against oxidative damage caused by ROS under such stresses as water deficit, high light or salt, and so on. This study provides a profile of the expression pattern 
of LsTMT under high light and drought stresses, which, together with the information about its sequence of deduced amino acids, revealed its possible function in plants' reaction against oxidative stress to some extent. However, there is still much to do to uncover the specific function of the isolated $L S T M T$ gene in tocopherol metabolism by transgenic or gene knock-down techniques. And its regulatory mechanism on the molecular level also needs to be researched in the future.

\section{ACKNOWLEDGMENTS}

Research supported by the National Basic Research Program of China (973 Program, \#2007CB108805) and Shanghai Leading Academic Discipline Project (\#B209).

\section{REFERENCES}

Abbasi AR, Hajirezaei M, Hofius D, Sonnewald U, et al. (2007). Specific roles of $\alpha$ - and $\gamma$-tocopherol in abiotic stress responses of transgenic tobacco. Plant Physiol. 143: 1720-1738.

Cho EA, Lee CA, Kim YS, Baek SH, et al. (2005). Expression of $\gamma$-tocopherol methyltransferase transgene improves tocopherol composition in lettuce (Latuca sativa L.). Mol. Cells 19: 16-22.

Collin VC, Eymery F, Genty B, Rey P, et al. (2008). Vitamin E is essential for the tolerance of Arabidopsis thaliana to metal-induced oxidative stress. Plant Cell Environ. 31: 244-257.

Geourjon C and Deléage G (1995). Significant improvements in protein secondary structure prediction by consensus prediction from multiple alignments. Comput. Appl. Biosci. 11: 681-684.

Guo J, Liu X, Chen S and Jin Z (2006). Overexpression of VTE1 from Arabidopsis resulting in high vitamin E accumulation and salt stress tolerance increase in tobacco plant. J. Appl. Environ. Biol. 12: 468-471.

Hare PD, Cress WA and Van Staden J (1998). Dissecting the roles of osmolyte accumulation during stress. Plant Cell Environ. 21: 535-553.

Havaux M, Eymery F, Porfirova S, Rey P, et al. (2005). Vitamin E protects against photoinhibition and photooxidative stress in Arabidopsis thaliana. Plant Cell 17: 3451-3469.

Igamberdiev AU and Hill RD (2004). Nitrate, NO and haemoglobin in plant adaptation to hypoxia: an alternative to classic fermentation pathways. J. Exp. Bot. 55: 2473-2482.

Kiffin R, Bandyopadhyay U and Cuervo AM (2006). Oxidative stress and autophagy. Antioxid. Redox. Signal. 8: 152-162.

Kim YJ, Seo HY, Park TI and Baek SH (2005). Enhanced biosynthesis of $\alpha$-tocopherol in transgenic soybean by introducing $\gamma$-TMT gene. J. Plant Biotechnol. 7: 203-209.

Lee BK, Kim SL, Kim KH and Yu SH (2008). Seed specific expression of perilla $\gamma$-tocopherol methyltransferase gene increases $\alpha$-tocopherol content in transgenic perilla (Perilla frutescens). Plant Cell Tissue Organ. Cult. 92: 47-54.

Munne-Bosch S, Schwarz K and Alegre L (1999). Enhanced formation of alpha-tocopherol and highly oxidized abietane diterpenes in water-stressed rosemary plants. Plant Physiol. 121: 1047-1052.

Noctor G (2006). Metabolic signaling in defence and stress: the central roles of soluble redox couples. Plant Cell Environ. 29: 409-425.

Seong ES, Ghimire BK, Goh EJ and Lim JD (2009). Overexpression of the $\gamma$-TMT gene in Codonopsis lanceolata. Biol. Plant 53: 631-636.

Van Eenennaam AL, Lincoln K, Durrett TP, Valentin HE, et al. (2003). Engineering vitamin E content: from Arabidopsis mutant to soy oil. Plant Cell 15: 3007-3019.

Yamaguchi-Shinozaki K and Shinozaki K (1994). A novel cis-acting element in an Arabidopsis gene is involved in responsiveness to drought, low-temperature, or high-salt stress. Plant Cell 6: 251-264.

Yusuf MA and Sarin NB (2007). Antioxidant value addition in human diets: genetic transformation of Brassica juncea with $\gamma$-TMT gene for increased $\alpha$-tocopherol content. Transgenic Res. 16: 109-113.

Yusuf MA, Kumar D, Rajwanshi R, Strasser RJ, et al. (2010). Overexpression of $\gamma$-tocopherol methyl transferase gene in transgenic Brassica juncea plants alleviates abiotic stress: physiological and chlorophyll a fluorescence measurements. Biochim. Biophys. Acta 1797: 1428-1438. 